with the worst cognitive impairment were more likely to have been hospitalized and have a longer hospital stay than those in the mild cognitive impairment and normal cognition groups. Conclusions: A project to train nurses to screen for dementia was successful in identifying undiagnosed cognitive impairment. Increasing cognitive impairment in older people with diabetes was associated with poor diabetes control, less specialized care, and more hospitalizations.

\section{P2-533 IMPACT OF MILD COGNITIVE IMPAIRMENT ON PATIENT AND CARE PARTNER PREFERENCES FOR CARDIOVASCULAR TREATMENT}

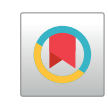

Bailey K. Reale ${ }^{1}$, Darin Zahuranec ${ }^{1}$, Kenneth M. Langa ${ }^{1}$, Jane Forman ${ }^{2}$, Bruno Giordani ${ }^{3}$, Brenda L. Plassman ${ }^{4}$, Kathleen A. Welsh-Bohmer ${ }^{4}$, Colleen Kollman ${ }^{5}$, Emilie Blair ${ }^{1}$, Deborah Levine ${ }^{1},{ }^{1}$ University of Michigan, Ann Arbor, MI, USA; ${ }^{2}$ VA Ann Arbor Healthcare System, Ann Arbor, MI, USA $;{ }^{3}$ Michigan Alzheimer's Disease Center, Ann Arbor, MI, USA; ${ }^{4}$ Bryan Alzheimer's Disease Research Center, Duke University, Durham, NC, USA; ${ }^{5}$ Kollman Research Services, Ann Arbor, MI, USA.

Contacte-mail: greenba@med.umich.edu

Background: The leading cause of death for the 5.4 million older adults with mild cognitive impairment (MCI) in the US is cardiovascular disease (CVD). Despite this fact, patients with pre-existing MCI may receive fewer treatments for CVD events such as acute myocardial infarction and acute ischemic stroke compared to cognitively normal patients. We conducted interviews of patients and care partners to understand how MCI influences preferences for stroke treatments. Methods: Qualitative study based on in-depth, semi-structured, in-person interviews with MCI patients and their care partners at 2 academic medical centers. We interviewed 23 dyads (MCI patient and care partner, $\mathrm{n}=11$; cognitively normal patient and care partner, $n=12$ ) using a standard interview guide. We used qualitative content analysis to identify unifying and recurrent themes. Using a clinical vignette, we elicited how MCI influences participant preferences for 5 common treatments for stroke: thrombolysis, feeding tube, inpatient rehabilitation, preventive medications, and carotid endarterectomy. We also asked participants to reflect on data from a pilot study suggesting that neurologists recommend fewer treatments for stroke to older adults with MCI. Results: Most MCI patients, cognitively normal patients, and their care partners wanted all 5 stroke treatments. They felt these treatments would minimize stroke effects, speed recovery, and prevent recurrent stroke (Table 1). Additionally, they thought that memory problems with MCI were not bad and that quality of life was still good. Participants reported several factors affecting their decision-making for treatment including treatment risks, alternative treatments, and physician recommendations (Table 1). Some participants thought that physicians might recommend fewer stroke treatments to patients with pre-existing MCI because physicians assume that MCI patients have poor prognosis or can't comply with treatment, discriminate against memory patients, or ignore patient and family preferences for care (Table 1). Other participants assumed that doctors' decisions were justified by data or experience. Conclusions: MCI patients have similar preferences for treatments for CVD events as cognitively normal patients do. We need to better understand how physician recommendations contribute to potential underuse of effective CVD treatments in MCI patients in order to improve the quality of CVD care for this large and growing population.

Table 1

Factors Influencing Decision-making for Stroke Treatments in Older Adults with Mild Cognitive Impairment (MCI)

Theme Supporting Quotes

Reasons Participants Want Stroke Treatments

To minimize stroke effects

- "The only thing I've heard about strokes is that you have to treat them right away and that there are dissolving medications that can help it go away and lessen the effects."

- "So, in order to minimize the overall effect."

To speed recovery

To prevent another stroke from happening

Memory problems with MCI not bad, quality of life still good

Alternative treatments

Physician recommendations

Factors that Influence Participant Preferences for Stroke Treatment Risks of treatment

Treatments for Patients with Pre-Existing MCI

Doctors assume MCI patients have poor prognosis

Doctors assume MCI patients can't comply with treatment

Doctors discriminate against memory patients
- "Maybe you would make faster improvement and strides toward, you know, your ADLs and everything "

- Preventing it would be easier than dealing with an additional stroke, 1 would think."

- "Well, to prevent 1 mean this is a catastrophic thing, depending on how much it affects the person, and to prevent it from happening again - for some reason it happened, so there could be a chance that it will happen again, so you want to guard against that."

- "I mean, it sounds like he's minimally involved with the memory, so the quality of life could still be very good if they could stop it."

- "I feel like there's more risk involved. Ifs also a lot more serious than, say. taking a medication. You're actually putting the person under, you're performing surgery, and there are risks - additional risks."

- "Were there medications that could open the blockage instead of surgery, that sort of thing."

• "I would talk to my doctor."

- "I would want to know, is that the cause of my stroke?"

- "Yeah. Just assuming they're older and don't heal as well"

- "Or are they just writing them off? "Well, they don't have a future."

- "Maybe they feel that the patient with mild memory problems might have more trouble remembering to take their medication."

- "That, plus, are they discriminating because it's a memory problem, they're going to have dementia. Alzheimer's, you know, they're not going to have a future?" 
Table 1

Factors Influencing Decision-making for Stroke Treatments in Older Adults with Mild Cognitive Impairment (MCI) (Continued)

\begin{tabular}{|c|c|}
\hline Theme & Supporting Quotes \\
\hline $\begin{array}{l}\text { Doctors ignore patient and family } \\
\text { preferences }\end{array}$ & $\begin{array}{l}\text { - "Never assume. Just discuss as } \\
\text { best you can to make that } \\
\text { determination. But never } \\
\text { assume." } \\
\text { • "It ticks me off because, again, } \\
\text { they're not treating the person as } \\
\text { being able to make any decisions } \\
\text { on their own personal care, and } \\
\text { that's not right." } \\
\text { "They need to discuss everything - } \\
\text { they should have those things } \\
\text { discussed with them along with } \\
\text { the caregiver so they all talk - You } \\
\text { can never assume that someone } \\
\text { doesn't understand what's being } \\
\text { told to them. Even if they have } \\
\text { dementia or other problems." }\end{array}$ \\
\hline
\end{tabular}

P2-534

\section{STATUS QUO OF HEALTH LITERACY IN INDIVIDUALS AT RISK FOR DEVELOPING ALZHEIMER'S DISEASE: FIRST DATA OF A SYSTEMATIC REVIEW}

Ayda Rostamzadeh $^{1}$, Julia Stapels ${ }^{1}$, Saskia Jünger ${ }^{2}$, Anna Genske ${ }^{3}$, Christiane Woopen ${ }^{2}$, Theresa Haidl ${ }^{1}$, Mauro Seves ${ }^{1}$, Kai Vogeley ${ }^{1}$, Frank Jessen ${ }^{1,4},{ }^{1}$ University Hospital of Cologne, Cologne, Germany; ${ }^{2}$ Ceres, University of Cologne, Cologne, Germany; ${ }^{3}$ Ceres, University of Cologne, Cologne, Germany; ${ }^{4}$ German Center for Neurodegenerative Diseases (DZNE), Bonn, Germany. Contact e-mail: ayda.rostamzadeh@ uk-koeln.de

Background: Health literacy (HL) can be described by specific skills that allow individuals to access, understand, appraise and apply information for decision-making and acting in health-related matters. In the field of early detection of Alzheimer's disease (AD), knowledge about HL in at risk individuals is limited. In the light of increasing technologies of early disease detection, risk assessment and prevention, individuals at risk are faced with complex information. This systematic review aims at analysing the status quo of empirical evidence on the role of health literacy for individuals at risk for developing AD. Methods: In the multiple-step search strategy, search terms and search strings were developed and pretested in PubMed. Search strings consisted of three sections, referring to $\mathrm{HL}, \mathrm{AD}$ and risk factors for developing AD. The search was carried out in PUBMED, Cochrane Library, PsycINFO and Web of Science. The complexity of the research question made it necessary to conduct a mixed-methods review, including both quantitative and qualitative study types. To be eligible, articles needed to report on empirical studies focusing on individuals at risk for developing $\mathrm{AD}$, and using either (a) a validated tool for assessing HL, or (b) mention the concept of HL as well as one of its four dimensions (access, understand, appraise, and apply). Results: A total of 3672 articles were identified and screened for eligibility by two independent reviewers. 211 articles were selected for full text review. None of the studies considered HL as a basic concept or used established HL assessment tools. However, 26 quantitative and 5 qualitative studies addressed at least one aspect of HL and were included for data extraction and analysis. Conclusions: This review reveals that systematic assessment of $\mathrm{HL}$ in an at-risk population for $\mathrm{AD}$ is sparse. Based on the outcomes of this review, qualitative interviews will investigate in more depth the meaning of HL for people at risk of developing AD. Eventually, the findings of this project will lay the foundation for the development of HL tools within the field of early $\mathrm{AD}$ diagnosis, and for interventional approaches to a competent handling of health-related risk information.

\section{P2-535 COMMUNICATION DIFFICULTIES: A SYNTHESIS OF EVIDENCE OF CURRENTLY AVAILABLE TOOLS USED TO ASSESS LANGUAGE AND COMMUNICATION IN DEMENTIA}

Luisa Krein ${ }^{1}$, Yun-Hee Jeon ${ }^{1}$, Amanda Miller-Amberber ${ }^{2}$, Judith Fethney ${ }^{1}$, ${ }^{1}$ University of Sydney, Sydney, Australia; ${ }^{2}$ Australian Catholic University, Sydney, Australia.Contacte-mail: yun-hee.jeon@sydney.edu.au

Background: Language and communication impairment (LCI) affect the vast majority of people with dementia at some stage. Accurate and timely assessment of LCI is vital for adequate management. However, no previous research has compared the psychometric properties and utility of currently available tools for the assessment of LCI in dementia. Methods: A broad scoping review of the literature was conducted to identify tools used for the assessment of language and communication in dementia from 1970 - 2017. To filter tools most appropriate for dementia populations, predefined eligibility criteria were applied, and 18 tools met these criteria. To identify the best tools, with rigorous psychometric properties and evidence for use in research and practice, a systematic search was conducted for each of the 18 tools and an objective ten-point scoring system was applied. The systematic search involved four major health databases and was complemented by a google and google scholar web search as well as by forward and backward citation searches. Results: Three tools received an objective score of ten points and were reviewed indepth. The Arizona Battery of Communication Disorders in Dementia may be best used to comprehensively inform clinical language and functional communication-related management in mild to moderate Alzheimer's disease. The Sydney Language Battery is best used for screening language in PPA populations and can help determine the PPA variant. The Addenbrooke's Cognitive Examination-III is the best instrument to gain a broad understanding of language impairment in the context of neuropsychological cognitive function testing. Conclusions: This review provides a synthesis of evidence for 18 tools commonly used for LCI-assessment in dementia. Three tools with the best combination of evidence regarding psychometric properties and best demonstrated utility in various clinical and research contexts are highlighted. Main limitations of the top tools revolve around limited standardization for dementia types other than Alzheimer's disease. Clinicians should be aware of the variety of instruments available and consider outlined characteristics when choosing the most appropriate tool for their assessment purpose. 